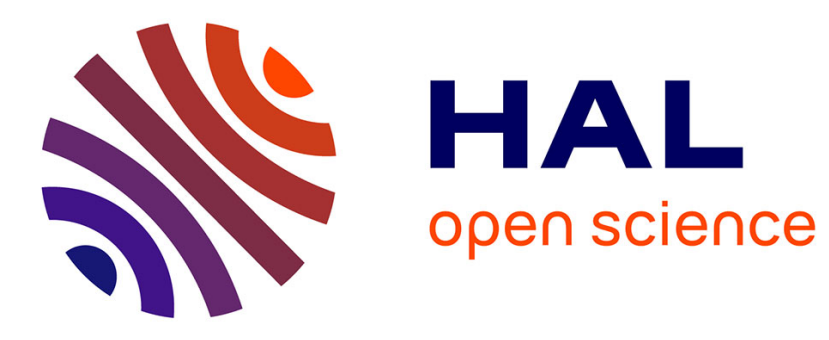

\title{
Modelling of a Flexible Quadritor Helicopter
}

\author{
Alexis Mouhingou, Naoufel Azouz
}

\section{To cite this version:}

Alexis Mouhingou, Naoufel Azouz. Modelling of a Flexible Quadritor Helicopter. ASME 2006 International Design Engineering Technical Conferences and Computers and Information in Engineering Conference, Sep 2006, Philadelphia, United States. pp.635-644, 10.1115/DETC2006-99001 . hal00342866

\section{HAL Id: hal-00342866 https://hal.science/hal-00342866}

Submitted on 7 Jan 2019

HAL is a multi-disciplinary open access archive for the deposit and dissemination of scientific research documents, whether they are published or not. The documents may come from teaching and research institutions in France or abroad, or from public or private research centers.
L'archive ouverte pluridisciplinaire HAL, est destinée au dépôt et à la diffusion de documents scientifiques de niveau recherche, publiés ou non, émanant des établissements d'enseignement et de recherche français ou étrangers, des laboratoires publics ou privés. 


\title{
MODELLING OF A FLEXIBLE QUADRITOR HELICOPTER
}

\author{
MOUHINGOU Alexis, AZOUZ Naoufel \\ Laboratoire Systèmes Complexes \\ Université d'Evry Val d'Essonne \\ 40, rue du Pelvoux, 91020 Evry cedex, France \\ E-mail : alexis.mouhingou, azouz@iup.univ-evry.fr
}

\begin{abstract}
This paper describes the dynamical modelling for the simulation of the quadrirotor Helicopter in order to see the influence of the flexibility on the dynamical model. We consider a quadrirotor Helicopter like a multibody systems constituted of the flexible and rigid substructures interconnected by of articulation joint. We use the variationnal Lagrangian approach to define the equations governing the motion. Deformations modes are used to represent elastic deformations of the substructure relative to a body reference frame. The displacement functions shape of the flexible components is obtained by a finite element discretization technique. The numerical application is related to the quadrirotor XSF developed in the LSC (Laboratory of Complex Systems)
\end{abstract}

Keywords: modelling, flexible multibody systems, Lagrangian dynamics, substructuration, UAV.

\section{Mathematical notations.}

$\left(R_{g}\right)=\left\{T, \vec{x}_{g}, \vec{y}_{g}, \vec{z}_{g}\right\}$ inertial frame.

$\left(R_{1}\right)=\left\{O_{1}, \vec{x}_{1}, \vec{y}_{1}, \vec{z}_{1}\right\}:$ is the local reference frame of the central body.

$\left(R_{i}^{*}\right)=\left\{O_{i} \vec{x}_{i}^{*}, \vec{y}_{i}^{*}, \vec{z}_{i}^{*}\right\}$ : the local reference frame of the undeformed flexible body.

$\left(R_{i}\right)=\left\{O_{i} \vec{x}_{i}, \vec{y}_{i}, \vec{z}_{i}\right\}$ the local reference frame of the flexible ( $i=$ fuselage, rotor-blades).

$\left(R_{i / j}^{p}\right)=\left\{P_{i / j} \vec{x}_{i / j}^{p}, \vec{y}_{i / j}^{p}, \vec{z}_{i / j}^{p}\right\} \quad$ local reference frame of the revolute joint point between fuselage and rotor- blades.

$\underline{x}_{i}$ : matrix $(n \times 1)$ of the components of the vector $\vec{X}^{i}$ expressed in the inertial frame.

$\underline{x}^{i}$ : matrix $(n \times 1)$ of the components of the vector

$\vec{X}^{i}$ express in the reference frame of body $i$.

$\underline{\underline{X}} \tilde{X}^{i}$ is the skew matrix associated to $\underline{x}^{i}$.

$\underline{X}$ : square matrix $(n \times n)$
This work is supported by the microflyer competition program organized by the DGA (Direction General des Armements) and the ONERA (Office National d'Etudes et de Recherches en Aerospatiale), France.

\section{Introduction}

The recent popularity of the Unmanned Aerial Vehicles (UAV), introduced a need for exploring new civil tasks such as search and rescue, surveillance and inspection. For the two last tasks, the rotary wing aerial vehicles have an important advantage over conventional fixed wings aircrafts because they can easily hover above the target. To execute some special tasks the UAV should also be small and autonomous. This is now possible, through the advancement of micro-mechanisms and microelectronics. For this purpose, we discuss in this paper the design, and modelling of a miniQuadrotor helicopter.

Current literature in the area of design of Quadrotor helicopters focuses on the development of indoor prototypes, see [6-9]. The design proposed is illustrated by Very light systems with fixed-rotors. However, the main problem associated with this concept is the evident instability of these prototypes in front of atmospheric perturbations especially when manoeuvring.

We propose in this study a suitable design of a quadrotor for outdoor applications with a large energetic autonomy, capable to avoid obstacles, having an embedded algorithm of stabilisation, and able to execute an automatic taking off and landing. This UAV can eventually be used by the foot soldiers to explore hostile villages.

To built the dynamic model we consider the quadrirotor helicopter as a flying flexible multibody system. There are many engineering examples that 
considered the flying objects as multibody systems. These mechanical systems can be presented in the form of rigid or flexible body. In both cases, it is important to define the coordinates system of motion. When describing the motion of rigid bodies in space, it is convenient to attach a set of axes to the body. It is quite common to describe the motion of rigid bodies in terms of the translation of the origin of the body axes and the rotation of the body axes; the corresponding variables, particulary the rotations, are referred to quasi-coordinates. The situation is complicated for flexible bodies, in which case there are basically two types of reference frames. First, the fixed one in the undeformed body describes the translation and the rotation motion of the origin of the reference frame fixed on the body axes. The second reference is the moving one relative to the undeformed body.

A suitable algorithm have been developed by [1-2]. They consider the slider crank mechanism as a flexible multibody systems, and developed an analytical technique based on a variationnal method which permits to use existing "rigid" codes for the dynamical simulation of this flexible slider crank.

The dynamical simulation of the mechanical systems with interconnected bodies presents an obvious difficulty in the joints that usually leads to non linear dynamical models. The non linearity of these systems is also introduced by gyroscopic effects and aerodynamic loads, especially when we study flying objects. To prevent this kind of problems in the dynamical simulation, several searchers use linear models. This is also important in order to have suitable models for the control and stability tasks. Recent works [7-10] present the quadrirotor as a material body without components.

We chose in this study to present a general lagrangian method that define the dynamical model of the quadrirotor assumed as a flying multibody system. The model should include the flexibility of the arms and blades and the non-linear effects. A modal synthesis is coupled to the algorithm for a suitable description of the deformation and its interaction with the whole motion.

\section{Presentation of the XSF Model}


dynamics of quadrirotor because of the presence of several elements. This substructuration method consists with the subdivision of the UAV in elementary bodies interconnected by kinematics joints such as shown in figure 2.

The substructures $\{5-8\}$ are a flexible arm, witch substructures $\{5\}$ and $\{7\}$ is connected at the central body by a fix joint. However $\{6\}$ and $\{8\}$ is connected by a revolute joint around the $\vec{x}_{1}$ axis. The substructure $\{1-4\}$ is the subsystem rotor-two blades, such as the rotor is the rigid body and the blades are a flexible body. At each free extremity of the arms $\{5\},\{6\},\{7\}$ and $\{8\}$ is connected the axis of the identique components $\{1-4\}$ by revolute joints around the $\vec{z}_{1}$ axis. The whole of the system is in spangled form with a central body. We considered that the multibody system of the Quadrirotor helicopter is composed by a four identical systems made up a flexible fuselage and a rotor-two flexible blades system.

Thus, initially we begin our study with the study of system fuselage-rotor-blades. To establish the dynamic equations of this system, In the first step, we establish the mathematical equations of the fuselage and the rotor-blade system separately. In the second step we take account the revolute joint between the fuselage and the rotor-blades system by a multipliers Lagrange technical.

To establish the complete dynamic equations of the XSF we deduct the dynamical of the others bodies by a symmetric properties such as that will be developed in the continuation.

\subsection{Kinematics of MBS}

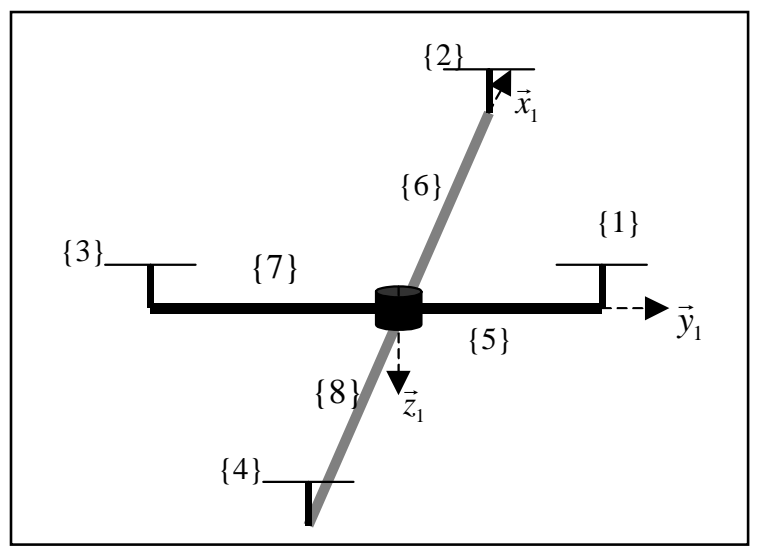

Figure 2. Kinematic scheme of MBS.

Let $O_{j}$ the origin of the local reference frame fixed at the $\{j\}$ body, the motion of the flexible $\{j\}$ body is subdivided into a rigid body motion and deformation as shown in figure 3. The location of an arbitrary point $P^{j}$ of body $\{j\}$ with respect to an inertial frame $\left(R_{0}\right)$ is given by:

$$
\underline{Y}_{i}\left(P_{i}\right)=\underline{R}_{i}\left(O_{i}\right)+\underline{\underline{A}}^{i T}\left(\underline{X}^{i}+\underline{u}^{i}\right)
$$

In this first section we neglected the $i$ index, thus the equation of motion of written as:

$$
\underline{Y}(P)=\underline{R}(O)+\underline{\underline{A}}^{T}(X+u)
$$

where:

$\underline{R}(O)$ : express the components of the translation vector such as:

$\underline{R}=\left\{\begin{array}{l}x_{0} \\ y_{0} \\ z_{0}\end{array}\right.$

$\underline{=}(t)$ : is the orientation matrix that defines the orientation of local reference frame fixed at the moving body with respect to the inertial reference frame

$$
\stackrel{\underline{\underline{A}}}{=}\left(\theta_{1}, \theta_{2}, \theta_{3}\right)=\left(\begin{array}{ccc}
c_{3} c_{2} & c_{3} s_{2} s_{1}-s_{3} c_{1} & c_{3} s_{2} c_{1}+s_{3} s_{1} \\
c_{3} S_{2} & S_{3} S_{2} s_{1}+c_{3} c_{1} & S_{3} s_{2} c_{1}-c_{3} s_{1} \\
-S_{2} & c_{2} s_{1} & c_{2} c_{1}
\end{array}\right)
$$

$$
\begin{aligned}
& c_{1}=\cos \varphi ; s_{1}=\sin \varphi \\
& c_{2}=\cos \theta ; s_{2}=\sin \theta \\
& c_{3}=\cos \psi ; s_{3}=\sin \psi \\
& \theta_{1}=\varphi ; \theta_{2}=\theta ; \theta_{3}=\psi \\
& (\varphi, \theta, \psi): \text { is Euler angle’s } \\
& X=\left\{\begin{array}{l}
x \\
\eta \\
\zeta
\end{array}\right.
\end{aligned}
$$

is the position vector of an arbitrary point $P$ pertaining to a section before the deformation of the body express in the local reference frame. We considered in the continuation that the body is assimilated at the beam. 


\subsection{Discretization of the beam}

We use the linear theory of the beam such as the flexibility displacement is only on the vertically direction i.e. $u_{3}(x, t)$ such as:

$u_{3}(x, t)=\sum_{n=1}^{\mu} \phi_{n}(x) q_{n}(t)$

given by a Rayleigh-Ritz discretization, using this formulation we written the flexible displacement vector as:

$u(x, t)=\left[\begin{array}{l}u_{1} \\ u_{2} \\ u_{3}\end{array}\right]=\sum_{n=1}^{\mu}\left[\begin{array}{c}-\frac{\zeta}{l} \frac{\partial \phi_{n}(\xi) q_{n}(t)}{\partial \xi} \\ 0 \\ \phi_{n}(\xi) q_{n}(t)\end{array}\right]$

$(\eta, \zeta)$ : is the coordinates of local section of the beam,

$\phi_{n}(x)$ : represent the spatial modal function define by an interpolation function that will be developed in the next section.

$q_{n}(t)$ : are the flexible characterized coordinates systems.

$l$ : the length of the flexible body

$x=\frac{\xi}{l}:$ is the position of the arbitrary point

In this work, we considered the flexible arm like a tubular flexible beam fixed with a mass with the end, the blades of the rotor are considered as a free fixed thin beam. The numerical values of the sharp modes selected are consigned in the following table such as:

\begin{tabular}{|l|l|l|}
\hline $\begin{array}{l}\text { Mode } \\
\text { retained }\end{array}$ & Flexible arm & $\begin{array}{l}\text { Rotor } \\
\text { propeller }\end{array}$ \\
\hline$k_{1}$ & 1.014 & 1.875 \\
\hline$k_{2}$ & 4.007 & 4.096 \\
\hline$k_{3}$ & 7.050 & 7.855 \\
\hline
\end{tabular}

The angular velocity vector $\vec{\Omega}$ of body can be expressed by:

$$
\vec{\Omega}=\dot{\psi} \vec{z}_{g}+\dot{\theta} \vec{y}_{i}^{*}+\dot{\phi} \vec{x}_{i}
$$

and we can easily define a relation between the angular velocity vector in the inertial frame $\underline{\Omega}$ and the time derivatives of Euler angles $\underline{\dot{\Theta}}$ such as:

(see [11]) where: $\quad \dot{\Theta}=\left[\begin{array}{lll}\dot{\phi} & \dot{\theta} & \dot{\psi}\end{array}\right]$

$\dot{\phi}, \dot{\theta}, \dot{\psi}$ are the derivatives of Euler angles.

The absolute velocity of each material point $P$ of body with respect to inertial frame is derived from Eq. (1) as [11]:

$$
\underline{V}=\underline{\dot{R}}+\underline{\underline{A}}(\underline{\underline{\tilde{X}}}+\underline{\underline{\tilde{u}}})^{T} \underline{\underline{G}} \underline{\dot{\Theta}}+\underline{\underline{A}} \dot{\underline{u}}
$$

\subsection{Kinetic energy}

The kinetic energy of flexible body can be written as:

$T=\frac{1}{2} \int_{(B)}(V)^{T}(V) d m$

$m$ : is the mass of the body

Let us consider the generalized position vector $\underline{\alpha}$ defined as: $\underline{\alpha}=\left[\begin{array}{lll}\underline{R} & \underline{\Theta} & q_{n}\end{array}\right]^{T}$

where $q_{n}$ represents the amplitude of deformation. The kinetic energy can then be written as:

$T=\frac{1}{2} \underline{\dot{\alpha}}^{T} \underline{\underline{M}} \underline{\dot{\alpha}}$

$\underline{\underline{M}}$ : is the $(6+n) \times(6+n)$ bloc-matrix of mass of the body, such as:

$\underline{\underline{M}}=\left[\begin{array}{ccc}\underline{\underline{M}}_{R R} & \underline{\underline{M}}_{R \Theta} & \underline{\underline{M}}_{R f} \\ \text { symmetric } & \underline{\underline{M}}_{\Theta \Theta} & \underline{\underline{M}}_{\Theta f} \\ & & \underline{\underline{M}}_{f f}\end{array}\right]$

When using the Eq. (5'), the kinetic energy terms can be written as such:

$\underline{\underline{M}}_{R R}=\underline{\underline{m}}$

$\underline{\underline{m}}=\left[\begin{array}{lll}m & & \\ & m & \\ & & m\end{array}\right]$

$\underline{\underline{M}}_{R \Theta}=\underline{\underline{A}}\left(\int_{(B)}(\underline{\underline{\tilde{X}}}+\underline{\underline{\tilde{u}}})^{T} d m\right) \underline{\underline{G}}$ 


$$
\begin{aligned}
& \underline{\underline{M}}_{R f}=\underline{\underline{A}} \int_{(B)} \underline{\underline{\phi_{n}}} d m \\
& \underline{\underline{M}}_{\Theta \Theta}=\underline{\underline{G}}^{T}\left(\int_{(B)}(\underline{\underline{\tilde{X}}}+\underline{\underline{\tilde{u}}})(\underline{\underline{\tilde{X}}}+\underline{\underline{\tilde{u}}})^{T} d m\right) \underline{\underline{G}} \\
& \underline{\underline{M}}_{\Theta f}=\underline{\underline{G}}^{T} \int_{(B)}(\underline{\underline{\tilde{X}}}+\underline{\underline{\tilde{u}}})^{T} \underline{\underline{\varphi}}_{n} d m \\
& \underline{\underline{M}}_{f f}=\int_{(B)}\left(\underline{\underline{\varphi}}_{n}\right)^{T}\left(\underline{\underline{\varphi}}_{n}\right) d m
\end{aligned}
$$

We consider the differents hypothesis on small displacement of the deformations modular and the length of the beam such as:

$$
\frac{u}{x} \ll 1,
$$$$
\Delta u \ll 1 \text {, }
$$

the expression of matrix mass become:

$$
\begin{aligned}
\underline{\underline{M}}_{R \Theta} & =\underline{\underline{A}}\left(\int_{(B)}(\underline{\underline{\tilde{X}}})^{T} d m\right) \underline{\underline{G}} \\
\underline{\underline{M}}_{\Theta \Theta} & =\underline{\underline{G}}^{T}\left(\int_{(B)}(\underline{\underline{\tilde{X}}})(\underline{\underline{\tilde{X}}})^{T} d m\right) \underline{\underline{G}} \\
\underline{\underline{M}}_{\Theta f} & =\underline{\underline{G}}^{T} \int_{(B)}(\underline{\underline{X}})^{T} \underline{\underline{\varphi}}_{n} d m
\end{aligned}
$$$$
\underline{\underline{M}}_{R \Theta}=\stackrel{A}{=}\left[\begin{array}{ccc}
0 & 0 & 0 \\
0 & 0 & \frac{1}{2} m l \\
0 & -\frac{1}{2} m l &
\end{array}\right] \underline{\underline{G}}
$$

where:

$$
\begin{aligned}
& b_{n}\left(k_{n}\right)=\frac{1}{k_{n}}\left(2+\sin \left(k_{n}\right)-\operatorname{sh}\left(k_{n}\right)-\cos \left(k_{n}\right)-\operatorname{ch}\left(k_{n}\right)\right) \\
& \underline{M}_{R f}=\underline{=}\left[\begin{array}{llll}
0 & 0 & \cdots & 0 \\
0 & 0 & \cdots & 0 \\
m b_{1}\left(k_{1}\right) & m b_{2}\left(k_{2}\right) & \cdots & m b_{\mu}\left(k_{\mu}\right)
\end{array}\right] \\
& \underline{\underline{M}}_{\Theta \Theta}=\underline{\underline{G}}^{T}\left[\begin{array}{ccc}
I_{x x} & 0 & 0 \\
0 & I_{y y} & 0 \\
0 & 0 & I_{z z}
\end{array}\right] \underline{\underline{G}}
\end{aligned}
$$

with:

$$
\begin{aligned}
& a_{n}=\left(\cos \left(k_{n}\right)+\operatorname{ch}\left(k_{n}\right)+\sin \left(k_{n}\right)-\operatorname{sh}\left(k_{n}\right)\right) \\
& d_{n}=\frac{1}{k_{n}^{2}}\left(\begin{array}{l}
\left(1-k_{n}\right)\left(\cos \left(k_{n}\right)+e^{k_{n}}\right)+ \\
\sin \left(k_{n}\right)\left(1+k_{n}\right)
\end{array}\right)
\end{aligned}
$$

$$
\begin{aligned}
& \underline{\underline{M}}_{\Theta f}=\underline{\underline{G}}^{T}\left[\begin{array}{cccc}
0 & 0 & \cdots & 0 \\
\left(a_{1}+d_{1}\right) & \left(a_{2}+d_{3}\right) & \cdots & \left(a_{\mu}+d_{\mu}\right) \\
0 & 0 & \cdots & 0
\end{array}\right] \\
& \underline{\underline{M}}_{f f}=\int_{(B)}\left(\phi_{n}\right)^{T}\left(\underline{\left.\underline{\phi_{n}}\right) d m}\right.
\end{aligned}
$$$$
\underline{\underline{M}}_{f f}=\left[\begin{array}{ccc}
m_{f_{11}} & \cdots & m_{f_{1 \mu}} \\
\vdots & \cdots & \vdots \\
m_{f_{\mu 1}} & \cdots & m_{f_{\mu \mu}}
\end{array}\right]
$$$$
m_{f_{\mu \mu}}=m_{d} \int_{0}^{1}\left(\phi_{\mu}^{\prime}(\xi) \phi_{\mu}^{\prime}(\xi)+\phi_{\mu}(\xi) \phi_{\mu}(\xi)\right) d \xi
$$

$m_{d}$ depends only on the dimensions and the mass of the beam.

The elastic potential energy could be expressed as:

$U=\frac{1}{2} \underline{\alpha}^{T}\left(\int_{(B)} E I\left(\underline{\underline{\varphi}}_{n}^{\prime \prime}\right)^{T}\left(\underline{\underline{\varphi}}_{n}^{\prime \prime}\right) d v\right) \underline{\alpha}$

where:

$v$ : express the volume of the body

$$
\begin{aligned}
& \varphi_{n_{k}}^{\prime \prime}(\xi)=\frac{1}{l^{2}} \frac{\partial^{2} \varphi_{n_{k}}(\xi)}{\partial \xi^{2}} \text { the elements of the } \\
& \underline{\underline{\varphi}}_{n}^{\prime \prime}(\xi) \text { matrix }
\end{aligned}
$$

One can write the Lagrangian of the motion of the flexible body as:

$$
\ell=T-U
$$

\subsection{Equation of Motion of the Body}

To generate the equation of motion of the body using the Lagrangian formalism, we use a variational method based on the principle of virtual works. We obtain the dynamic equation in this form:

$\underline{\underline{M}} \underline{\underline{\alpha}}+\underline{\underline{C}} \underline{\dot{\alpha}}+\underline{K} \underline{\alpha}=\underline{Q}_{e}$

The mass Matrix $\underline{\underline{M}}$ contains the inertial terms in translation, rotation and deformation motion.

$\underline{\underline{C}}$ is the $(6+n) \times(6+n)$ matrix of gyroscopic terms as such: 


$$
\begin{aligned}
& \underline{\underline{C}}=\left[\begin{array}{ccc}
\underline{\underline{M}}_{R R} & \dot{\underline{M}}_{R \Theta} & \dot{\underline{M}}_{R f} \\
\underline{\bar{M}}_{R \Theta}^{T} & \overline{\bar{M}}_{\Theta \Theta} & \underline{\bar{M}}_{\Theta f} \\
\underline{\underline{M}}_{R f}^{T} & \underline{\underline{M}}_{\Theta f}^{T} & \underline{\underline{M}}_{f f}
\end{array}\right] \\
& \underline{\underline{M}}_{k k}=\frac{d\left(\underline{\underline{M}}_{k k}\right)}{d t}, k=R, \Theta, f
\end{aligned}
$$

$\underline{\underline{K}}$ is the stiffness matrix of the body

$$
\begin{aligned}
& \underline{\underline{K}}=\left[\begin{array}{ccc}
0 & 0 & 0 \\
& 0 & 0 \\
\text { symétric } & & \underline{K}_{f f}
\end{array}\right] . \\
& \underline{K}_{f f}=\left(\int_{(B)} E I\left(\underline{\underline{\varphi}}_{n}^{\prime \prime}\right)^{T}\left(\underline{\underline{\varphi}}_{n}^{\prime \prime}\right) d v\right)
\end{aligned}
$$

$Q_{e}$ : the generalized force vector acting on the body that we will developed in the next section.

\subsection{Equation of motion of the Quadrirotor helicopter.}

Before to write the equation of motion of the Quadrirotor helicopter (XSF-4), we consider the joint linking different components of the XSF-4 assimilated to a flexible multibody system. In this case of XSF-4 the principle joint between a components i.e. fuselage and rotor-blades is a revolute joint.

\subsubsection{Modelling of the revolute joints}

The modelling of Multibody system requires the integration of the kinematic joints due to the interconnections of the bodies. The kinematic constraints of the joint between the body $\{i\}$ and body $\{j\}$ are given by:

$$
H^{j}\left(\underline{\alpha}^{j}, t\right)-H^{i}\left(\underline{\alpha}^{i}, t\right)=0
$$

$H^{j}\left(\underline{\alpha}^{j}, t\right)$ : express the equations of the kinematic constraints of joints that define the constraint of translation and orientation of the points linked by the joint. We define this equation in the following section for each specific joint.

The links considered in this modelling are mainly the revolution joint between the arms and the rotorblades such as represented in figure 4 and the revolute joint between the substructure $\{5\}$ and the arm $\{6\}$ such as represented in figure 6 .

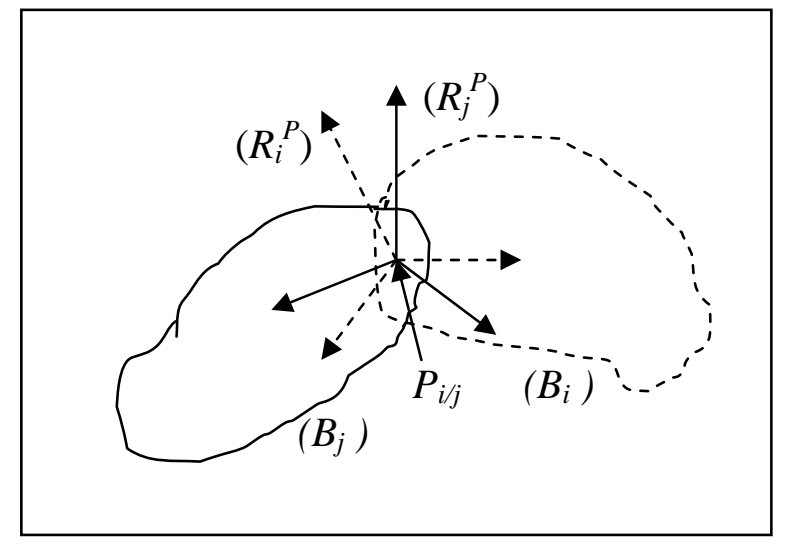

Figure 2. Representation of the revolute link between body $\{i\}$ and body $\{j\}$.

$P$ a point of link between flexible arm and rigid rotor.

Body $\{j\}$ is for example flexible arm and $\left(R_{j}^{P}\right)$ is its articulation reference frame of body $\{j\}$.

Body $\{j\}$ is for example a rotor blade and $\left(R_{i}^{P}\right)$ is its local reference frame.

\subsubsection{Equation of kinematic joint}

1. The equations of the kinematics constraints due with the blocking of the translation at the point $P_{i / j}$ of the articulation between body $\{i\}$ and body $\{j\}$ is given by a following equations:

$$
\left\{\begin{array}{c}
x_{0}^{i}+l\left(a_{11}^{i}-a_{21}^{i}\right)+\phi_{n_{i}}(\xi) q_{n_{i}}(t) a_{13}^{i}=x_{0}^{j}+a_{13}^{j} h \\
y_{0}^{i}+l\left(a_{11}^{i}+a_{21}^{i}\right)+\phi_{n_{i}}(\xi) q_{n_{i}}(t) a_{23}^{i}=y_{0}^{j}+a_{23}^{j} h \\
z_{0}^{i}+l a_{31}^{i}+\phi_{n_{i}}(\xi) q_{n_{i}}(t) a_{33}^{i}=z_{0}^{j}+a_{33}^{j} h
\end{array}\right.
$$

2. Let $\left(\vec{x}_{i}^{p}, \vec{y}_{i}^{p}, \vec{z}_{i}^{p}\right)$ and $\left(\vec{x}_{j}^{p}, \vec{y}_{j}^{p}, \vec{z}_{j}^{p}\right)$ : the unitary vectors of the articulation reference frame $\left(R_{i}^{P}\right)$ of body $\{i\}$ and $\left(R_{j}^{P}\right)$ of body $\{j\}$.The revolute joint satisfy the following condition such as: $\vec{Z}_{i}^{p}=\vec{Z}_{j}^{p}$

$\left(\vec{x}_{i}^{p}, \vec{y}_{i}^{p}, \vec{z}_{j}^{p}\right)$ Constitutes then a direct reference trihedral of the articulation $\{i-j\}$,

The mathematical equation of this condition such as:

$$
\left\{\begin{array}{l}
\vec{x}_{i}^{p} \cdot \vec{z}_{j}^{p}=0 \\
\vec{y}_{i}^{p} \cdot \vec{z}_{j}^{p}=0
\end{array}\right.
$$




$$
\left\{\begin{array}{l}
\left(a_{11}^{i}-a_{21}^{i}\right) a_{13}^{j}+\left(a_{11}^{i}+a_{21}^{i}\right) a_{23}^{j}+a_{31}^{i} a_{33}^{j}=0 \\
\left(a_{12}^{i}-a_{22}^{i}\right) a_{13}^{j}+\left(a_{12}^{i}+a_{22}^{i}\right) a_{23}^{j}+a_{32}^{i} a_{33}^{j}=0
\end{array}\right.
$$

where:

$\underline{A}^{i}=\left[\begin{array}{lll}a_{11}^{i} & a_{12}^{i} & a_{13}^{i} \\ a_{21}^{i} & a_{22}^{i} & a_{23}^{i} \\ a_{31}^{i} & a_{32}^{i} & a_{33}^{i}\end{array}\right]:$ is the orientation matrix function of the Euler angles.

$\Phi^{i / j}$ of the flexible body $\{i\}$ connected to the body $\{j\}\left(n_{i}=0\right.$ for the rigid body).

l: the length of the arm

$h$ : the long of the rotor

We use the kinematic equation to express the Jacobian matrix of Multibody System such as:

We consider the different revolute joint between the components of the multibody systems (Quadrirotor helicopter),

1. articulation of body $\{1\}$ and body $\{5\}$

2. articulation of body $\{2\}$ and body $\{6\}$

3. articulation of body $\{3\}$ and body $\{7\}$

4. articulation of body $\{4\}$ and body $\{8\}$

$$
\Phi_{j / i}=\left[\begin{array}{cccccc}
1 & 0 & 0 & h \frac{\partial a_{13}^{j}}{\partial \varphi_{j}} & h \frac{\partial a_{13}^{j}}{\partial \theta_{j}} & h \frac{\partial a_{13}^{j}}{\partial \psi_{j}} \\
0 & 1 & 0 & h \frac{\partial a_{23}^{j}}{\partial \varphi_{j}} & h \frac{\partial a_{23}^{j}}{\partial \theta_{j}} & h \frac{\partial a_{23}^{j}}{\partial \psi_{j}} \\
0 & 0 & 1 & h \frac{\partial a_{33}^{j}}{\partial \varphi_{j}} & h \frac{\partial a_{33}^{j}}{\partial \theta_{j}} & h \frac{\partial a_{33}^{j}}{\partial \psi_{j}} \\
0 & 0 & 0 & \frac{\partial\left(\vec{x}_{i}^{p} \cdot \vec{z}_{j}^{p}\right)}{\partial \varphi_{j}} & \frac{\partial\left(\vec{x}_{i}^{p} \cdot \vec{z}_{j}^{p}\right)}{\partial \theta_{j}} & \frac{\partial\left(\vec{x}_{i}^{p} \cdot \vec{z}_{j}^{p}\right)}{\partial \psi_{j}} \\
0 & 0 & 0 & \frac{\partial\left(\vec{y}_{i}^{p} \cdot \vec{z}_{j}^{p}\right)}{\partial \varphi_{j}} & \frac{\partial\left(\vec{y}_{i}^{p} \cdot \vec{z}_{j}^{p}\right)}{\partial \theta_{j}} & \frac{\partial\left(\vec{y}_{i}^{p} \cdot \vec{z}_{j}^{p}\right)}{\partial \psi_{j}}
\end{array}\right]
$$

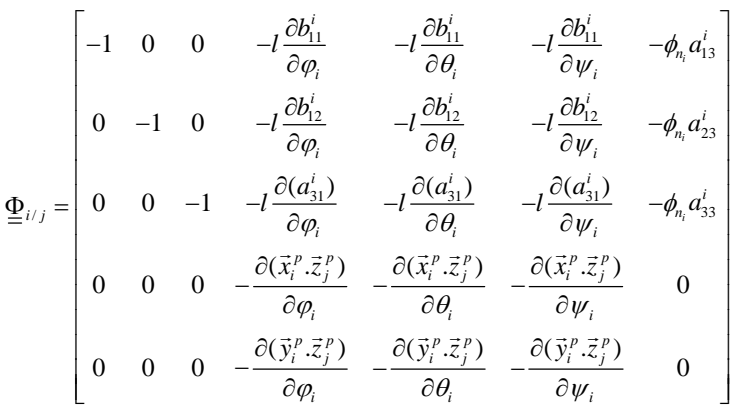

The compact form of the Jacobian matrix of multibody systems is given by:

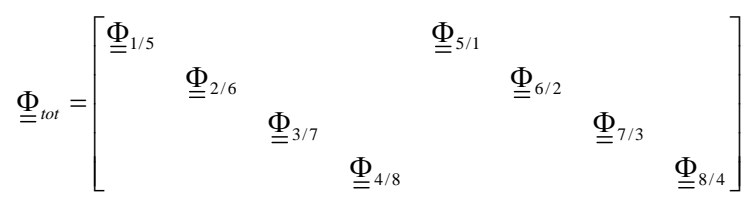

The equation of motion of the whole interconnected system is given by this expression:

\subsection{Generalized forces}

Let us assume that the location of a material point of the body is expressed by Eq. (1) and that the chosen virtual location is defined by:

$$
\delta \underline{Y}=\delta \underline{R}+\underline{\underline{A}}(\underline{\underline{\tilde{X}}}) \underline{\underline{G}} \delta \underline{\Theta}+\underline{\underline{A}} \underline{\underline{\varphi_{n}}} \delta q_{n}
$$

It results in the following expression for the virtual work of the applied forces:

$\delta W=(\underline{F})^{T} \delta \underline{Y} v$

The total force acting on the Quadrirotor is:

$\underline{F}=\rho \underline{g}+\underline{A}\left(\underline{F}_{L}+\underline{F}_{D}\right)$

where:

$\underline{F}_{L}=\sum_{i=1}^{4} F_{L} \cdot \underline{z}_{L}$ : express the aerodynamics forces vector in the blade local reference frame.

$\underline{F}_{D}=\sum_{i=1}^{4} F_{D} \cdot \underline{x}_{D}$ : express the drag force vector in the blade local reference frame

$\underline{g}:$ is the gravity acceleration vector

$\rho:$ is the masse density of the $j$ body $v:$ is the volume of body

$$
\underline{Q}_{R}^{T}=(\underline{F})^{T} v
$$


$\underline{Q}_{\theta}^{T}=(\underline{F})^{T} \underline{\underline{A}}(\underline{\underline{\tilde{X}}}) \underline{\underline{G}} v$

$\underline{Q}_{f}^{T}=(\underline{F})^{T} \stackrel{A}{=} \underline{\underline{\varphi}}=$

We obtain the corresponding expression of generalized forces vector acting on the Quadrirotor helicopter

$$
\underline{Q}_{e}^{T}\left[\begin{array}{lll}
\underline{Q}_{R} & \underline{Q}_{\theta} & \underline{Q}_{f}
\end{array}\right]^{T}
$$

\subsection{Aerodynamic forces and torques}

In this part, we will define the characteristics of the aerodynamic forces and torques issued from the blade theory.

The blade behaves as a rotating wing. Each element of the blade dr. is in contact with the airflow with a speed $V_{R}$ and according to an angle of attack $\beta$. One call pans the axisymmetric hooding of the hub, interdependent of the propeller in rotation. In the plan of the propeller, the pan is defined by the radius $S_{P}$.

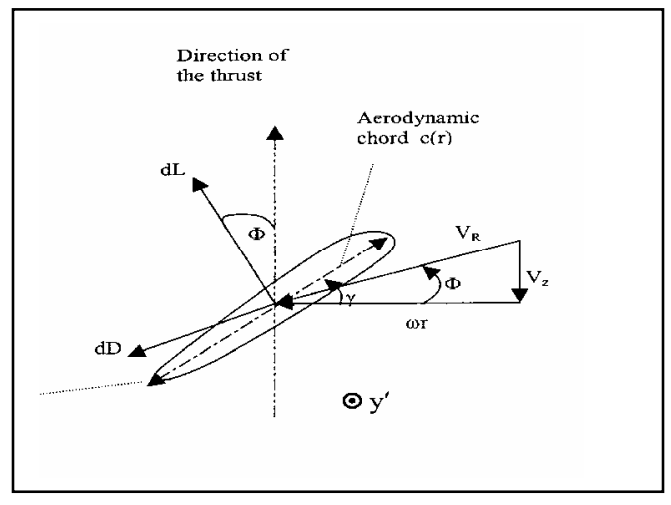

Figure 3. Description of forces applied on the blade.

Each elementary section of the blade of width $d r$ creates a lift $d L$ and a drag $d D$ [11], [12], such as:

$$
\left\{\begin{array}{l}
d L=\frac{1}{2} \rho_{\text {air }} C_{L} V_{R}^{2} d S \\
d D=\frac{1}{2} \rho_{a i r} C_{D} V_{R}^{2} d S
\end{array}\right.
$$

where $\rho_{\text {air }}$ is the air density, $C_{L}$ and $C_{D}$ represent adimensional coefficients of lift and drag depending mainly on the angle of attack $\beta$. $(\beta=\gamma-\Phi)$.
The XSF will hover or move at low speed, we can assume that the thrust $\bar{F}$ for a $B$ blades propeller can be written as:

$$
\left\{\begin{array}{l}
F_{L}=\frac{B}{2} \rho_{a i r} \omega^{2} \int_{0}^{S_{P}} r^{2} c(r) C_{L}(r) d r \\
F_{D}=\frac{B}{2} \rho_{a i r} \omega^{2} \int_{0}^{S_{P}} r^{2} c(r) C_{L}(r) d r
\end{array}\right.
$$

Or simply: $\left\{\begin{array}{l}F_{L}=k_{L} \omega^{2} \\ F_{D}=k_{D} \omega^{2}\end{array}\right.$

$k_{L}$ and $k_{D}$ are the coefficient of bearing pressure.

The computation of the lift coefficient is often complex. Moreover marginal swirls at the tip of the blade can modify significantly the theoretic values of some aerodynamic parameters.

It is thus essential to elaborate an experimental process, which will enable us to determine precisely the coefficient $k_{L}$ as well as the limits of validity of the equation (16).

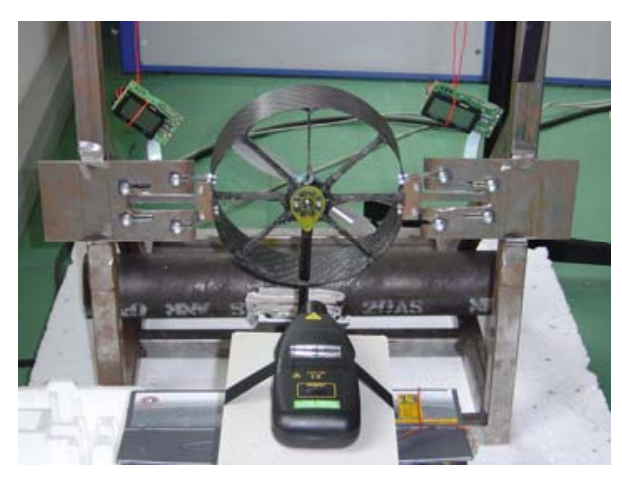

Figure 4. Testing bench of the thrust.

Let us denote $\overline{\bar{I}}_{R} \omega$ the kinetic moment of the rotor. The gyroscopic effects of the rotating elements are introduced in the model as follows:

$$
M_{G y}=\overline{\bar{I}}_{R} \omega \wedge \Omega
$$

These gyroscopic effects appear especially if an air disturbance create a change in roll or pitch angle, and when we swivel the arm $\{5\}$ and $\{6\}$. The gyroscopic moments are introduced in the global model (13) as generalised forces. 


$$
\left[\begin{array}{cc}
M_{t o t} & \Phi_{t o t}^{T} \\
\Phi_{\text {tot }} & 0
\end{array}\right]\left\{\begin{array}{l}
\ddot{\ddot{\alpha}}_{t o t} \\
\underline{\lambda}_{t o t}
\end{array}\right\}=\left\{\begin{array}{c}
\underline{Q}_{v}^{t o t}+\underline{Q}_{e}^{t o t} \\
\underline{Q}_{d}^{t o t}
\end{array}\right\}
$$

where:

$$
\underline{\underline{M}}_{t o t}=\left[\begin{array}{llll}
\underline{\underline{M^{1}}} & & & \\
& \underline{\underline{M^{2}}} & & \\
& & \ddots & \\
& & & \underline{\underline{M^{8}}}
\end{array}\right]
$$

is the bloc-diagonal mass matrix of all the bodies representing the Multibody system,

$\underline{\ddot{\alpha}}_{t o t}{ }^{T}=\left[\begin{array}{llll}\ddot{\alpha}^{1} & \ddot{\alpha}^{2} & \cdots & \ddot{\ddot{\alpha}}^{8}\end{array}\right]^{T}$ is the acceleration vector of all bodies, $\underline{\lambda}$ tot is the Lagrange multipliers vector, $\underline{Q}_{v}, \underline{Q}_{e}$ and $\underline{Q}_{d}$ are respectively centrifugal and gyroscopic, external and articulation generalised forces expressed in the inertial frame.

\section{Simulation and numerical Results}

The XSF is intended to move in an urban environment. Three kinds of manoeuvres are privileged: yaw rotation, vertical ascension, and translational displacement along the $\vec{x}_{1}$ axis.

In the first numerical test, we present the simulation of a vertical motion. Two models are considered. In the beginning we make the assumption that the XSF is a rigid body, subjected to external and gravity forces, and we compare the results with those of the full Lagrangian flexible model. The external forces applied on the XSF are gravity force and aerodynamic forces, which are function of the rotation speed of the rotorblades (Eq. 16), here:

$\omega=607.37 \mathrm{rad} / \mathrm{s}$,

$M=2.2 \mathrm{~kg}$ is the mass of the UAV,

$l=0.23 \mathrm{~m}$ the length of each arm.

$E=1.15 \times 10^{11} \mathrm{~Pa}$ Young modulus

$n_{i}=2$ : shape mode retained for each flexible body

The numerical simulation is based on the "semi explicit stable" Newmark method and developed in MATLAB $^{\circledR}$.

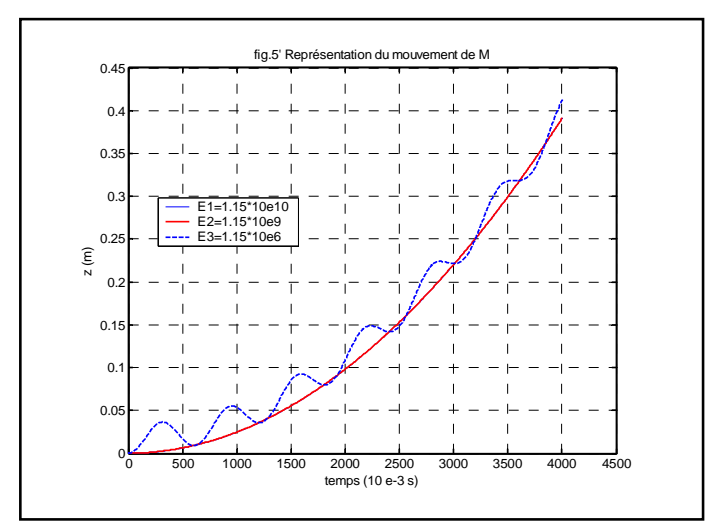

Figure 5. Comparison of global motion.

The figure 5 shows the global displacement of the tip of the arm $\{5\}$. The blue-dashed line shows the influence of the flexibility in comparison with the same arm supposed rigid in a hand and with the rigid Newton-Euler model developed in [8] in the other hand.

In the second test we simulate a full manoeuvre of the XSF in an urban environment. In the beginning it should rise to an altitude of $1.55 \mathrm{~m}$ corresponding roughly to its cruise altitude when exploring villages. Then we swivel the rotors (1) and (3) to permit the horizontal displacement along the x-axis. After that we impose a "square" trajectory composed by four quarter of turn followed by horizontal displacements. The different motions were controlled by a sample P.I.D. law.

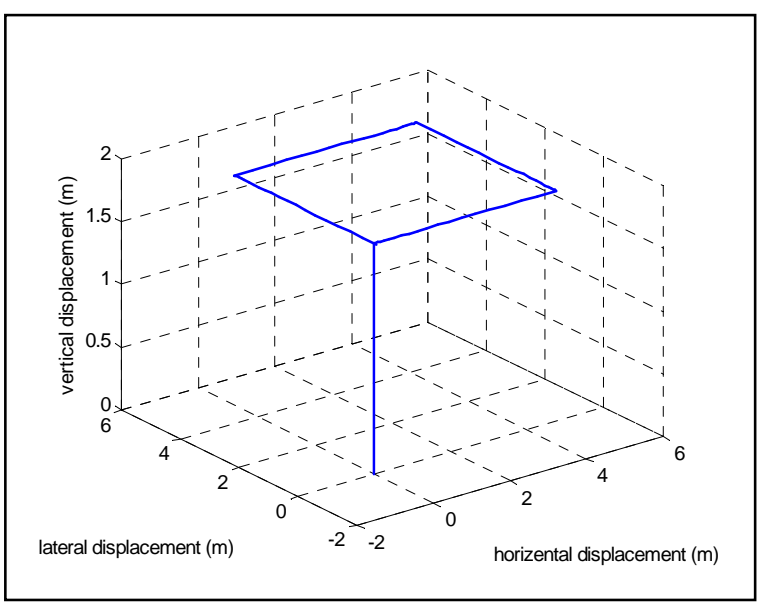

Figure 6. Trajectory of the XSF in urban mission.

The figure 6 shows the evolution of the position of the XSF centre of gravity in this mission. In a first approach we do not optimise the transition between one kinds of motion to another. This will be benefit to minimise the used energy. 


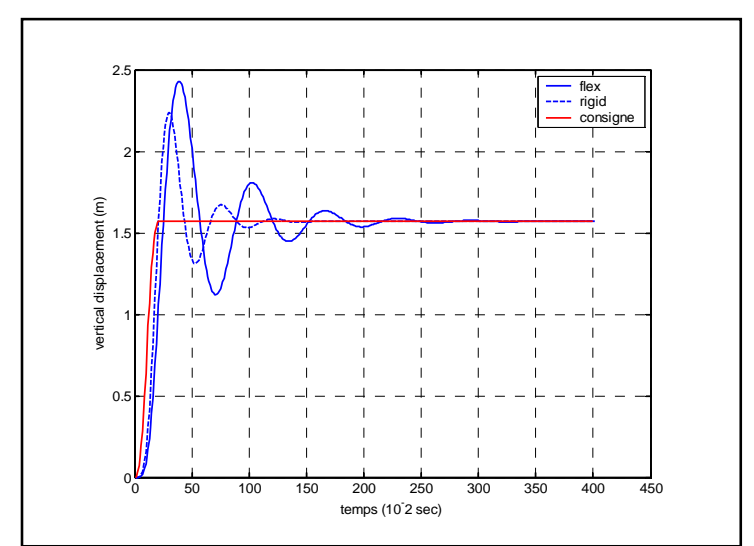

Figure 7. Flexible vertical displacement of XSF.

In figure 7 we show the vertical displacement of the tip of an arm in the ascendant phase and a comparison with the desired motion given by the PID law.

We compare in this figure the motion of the XSF when assumed rigid, and the one when we introduce the flexibility effect.

\section{Conclusion and future works}

The equations of motion for manoeuvring flying structure in urban environment are non-linear due to the large body motion and the flexibility of some components. The model presented in this paper takes into account this non-linearity and includes the effects of flexibility, and the aerodynamic and gyroscopic effects. The model shows the influence of the flexibility in reference to total rigid body models widely used in this field. This model will be completed later by the introduction of the aeroelasticity at the blades.

\section{Acknowledgement}

Authors want to thanks the graduate students Valentin Vala, Seneina Billel, Duaux Julien and Da Silva David for their help in the design and fabrication of the XSF, otherwise this project could not be achieved.

\section{References}

[1] PASCAL M. GAGARINA T. "A Pseudo-Rigid Model for the Dynamical Simulation of Flexible Mechanisms”, Multibody Systems Dynamics 3: 303-331, 1999
[2] PASCAL M. "Some Open Problems in Dynamic Analysis of Flexible Multibody Systems”, Multibody Systems Dynamics 5: 315-334, 2001

[3] BOYER F., COIFFET Ph. "Generalization of Newton-Euler model for flexible manipulators”, Int. Jour. For Robotics Research 17(3), pp. 282-293 (1996).

[4] SHABANA A.A. "Substructure synthesis methods for dynamic analysis of multibody systems ». Comp. \& Struct. Vol. 20 - n² 4 . pp. 737-744 (1985).

[5] SIMO J.C. " The role of non-linear theories in transient dynamic analysis of flexible structures ». Jour. of Sound and Vibr. 119, pp. 487-508 (1987).

[6] MARTI S. "The zero G eye: Towards a free hovering camera”. Technical Report, MIT, Massachusetts, USA (2000).

[7] CASTILLO P., DZUL A., LOZANO R., "Real-time stabilization and tracking of a four rotor Mini-Rotorcraft”. IEEE Transactions on Control Systems Technology, Regular paper, Vol.12, Issue 4, pp. 510-516, (2004).

[8] ALTUG E., OSTROWSKI J., MAHONY R., "Control of a quadrirotor helicopter using visual feedback". Proceedings of the IEEE Int. Conference on Robotics and Automation, ICRA ‘02, Washington DC, USA (2002).

[9] BOUABDALLAH S., SIEGWART R. "Backstepping and sliding-mode techniques applied to an Indoor quadrirotor”. Proceedings of the IEEE Int. Conference on robotics and automation, Barcelona, Spain (2005).

[10] AZOUZ N., BESTAOUI Y. "Modelling and simulation of a mini quadrirotor helicopter". Submitted to Journal of Aerospace Computing, Information, and Communication.

[11] SHABANA "Dynamics of Multibody systems”. Ed. Wiley, New York, (1998).

[12] GERADIN M., CARDONA A. "Flexible Multibody Dynamics: A Finite Element Approach "New York, USA (2001).

[13] BARNES W. McCormick, Jr. “Aerodynamics of V/STOL Flight” Darcorp, USA (1999).

[14] PADFIELD G. D. "Helicopter Flight Dynamics”. Blackwell Science. USA (1996). 